\title{
SOBREVIDA, MELD-SÓDIO E SÓDIO SÉRICO PÓS-TRANSPLANTE HEPÁTICO
}

\author{
Survival, Meld-Sodium and Serum Sodium Post-Liver Transplantation
}

\author{
Carolina Oliveira de Paulo', Luize Kremer Gamba', Alice Ferreira da Silva², Carla Martinez Menini-Stalhschmidt², \\ João Eduardo Leal Nicoluzzi², Matheus Takahashi Garciaª
}

\section{RESUMO}

Introdução: O Brasil é o maior sistema público de transplantes do mundo. Recentemente, o escore MELD-Sódio foi incorporado no Brasil como modelo de preditor de gravidade de doença hepática. Em estudos internacionais, foi observado que o sódio sérico abaixo dos valores normais está associado à perda do enxerto; no entanto, sua relação com fatores prognósticos na sobrevida pós-operatória ainda é incerta. Objetivos: Contribuir com a implementação de estratégias de rastreio de fatores prognósticos no pós-transplante hepático. Métodos: Estudo longitudinal retrospectivo, incluindo pacientes que realizaram transplante hepático no Hospital Angelina Caron, entre as datas de 01/01/2016 e 31/12/17 e que realizaram acompanhamento médico durante um ano após a data do transplante. Foram analisadas diferentes variáveis para estimar a taxa de sobrevida e fatores prognósticos pós-transplante. Resultados: Amostra final de 148 pacientes, dos quais $n=105$ pertenciam ao sexo masculino, com média de idade de 52 anos $\pm 11,7$, e n=139 autodeclararam-se de cor branca. Encontraram-se $n=50$ indivíduos com cirrose alcoólica e $n=32 \mathrm{com}$ hepatite viral. $\mathrm{O}$ tempo na lista de espera para o transplante hepático foi de 68 dias \pm 113 . A pontuação média do MELD foi de 20,7 $\pm 5,56$ e MELD-Na de 13,8 $\pm 5,31$. Encontrados valores MELD-Na $<15(n=105)$, MELD-Na $>15(n=43)$. A taxa de sobrevida geral pós-seis meses foi de $68,9 \%$, de 61,4\%, em 12 meses, e de $56,7 \%$, em 24 meses. Hiponatremia pré-operatória (<135 mEq/L) presente em 33 pacientes, dos quais $n=14$ vieram a óbito seis meses pós-transplante, $n=4$, em 12 meses, e $n=1$, em 24 meses. Hiponatremia e óbito obtiveram $p<0,05$. MELD-Na $>15(n=43)$, dos quais, 17 vieram a óbito seis meses pós-transplante, três, em 12 meses e um, em 24 meses. Avaliando o escore MELD, n=89 foram preditores significativos de óbito $(p<0,05)$, ajustando-o para o escore MELD-Na, $n=43$ foram preditores significativos de óbito $(p<0,05)$. Conclusão: A vigilância da hiponatremia pré-operatória e o uso do MELD-Na para definição da prioridade na lista de espera do procedimento devem ser fortemente incentivados como forma de redução da taxa de mortalidade pós-transplante hepático.

Descritores: Transplante de fígado, Hiponatremia, Sobrevida, Mortalidade, Prognóstico

Instituições:

${ }^{1}$ Pontifícia Universidade Católica do Paraná, Curitiba/PR, Brasil

${ }^{2}$ Hospital Angelina Caron, Campina Grande do Sul/PR, Brasil

\section{Correspondência:}

Nome: Carolina Oliveira de Paulo

Endereço: Av. Presidente Affonso Camargo, 881, Curitiba/PR-Brasil.

Telefone: (34) 99100-3663

E-mail: loracoliveira@hotmail.com

\section{INTRODUÇÃO}

O transplante de fígado é utilizado como alternativa viável a doenças hepáticas terminais, desde a década de 80. ${ }^{1}$ Atualmente, é considerado o procedimento mais complexo da cirurgia moderna, pois nenhum outro interfere com tantas funções do organismo. ${ }^{2}$

As indicações para o transplante incluem cirrose por hepatite, cirrose biliar, pacientes portadores de doenças hepatocelulares e colestáticas crônicas, doenças hepáticas metabólicas e vasculares, tumor primário do fígado e trauma. ${ }^{3,4}$

O Brasil é o maior sistema público de transplantes do mundo, com quase totalidade dos transplantes de 
órgãos feitos pelo Sistema Único de Saúde (SUS) - taxa próxima a $87 \%$. Em 2016, o número de transplantes de fígado bateu recorde no país, com 1.880 procedimentos, com uma fila de espera de 1.939 pacientes no mesmo período.

Diversos escores médicos são usados como modelos de prognóstico de gravidade da doença hepática na literatura médica. Eles estimulam a adoção de critérios de gravidade para alocação de enxertos, priorizando pacientes com menor expectativa de sobrevida. ${ }^{5}$

A taxa de sobrevivência média, após um ano de transplante hepático com doador falecido, em pacientes adultos, é superior a $85 \%$. Poucos relatos na literatura analisam quais fatores contribuem para a falha do enxerto hepático nos três primeiros meses pós-transplante. ${ }^{6}$

Criado nos anos 2000 e validado no Brasil em 2006, a escala de MELD (Model End-Stage Liver Disease Modelo para Doença Hepática Terminal), foi descrita, inicialmente, para estimar a sobrevida de pacientes submetidos a desvio venoso portassistêmico intrahepático transjugular (TIPS). Tem como variáveis os níveis séricos de creatinina, bilirrubina total e a razão internacional normalizada para o tempo de protrombina $(\mathrm{RNI})$ e a realização de hemodiálise na última semana. ${ }^{5,7}$

Em 2016, o MELD Na (MELD Sódio) substituiu o escore MELD tradicional na UNOS (United Network for Organ Sharing). Essa decisão foi feita para aprimorar o acesso dos pacientes aos transplantes e reduzir a mortalidade na lista de espera pré-transplante. A fórmula preconizada para o MELD-Na é: MELD-Na = MELD + 1.32 X (137 $X \mathrm{Na})[0,033 \times$ MELD $\times(137-\mathrm{Na})] .^{8} \quad$ No ano de 2019, o escore MELD-Na foi incorporado pela Associação Brasileira de Transplantes de Órgãos (ABTO) como critério de seleção de receptores na lista de espera para transplante hepático.

Foi visto que a natremia está associada significantemente à disfunção precoce do enxerto, isto é, quanto menor o valor do sódio sérico, maior a probabilidade do paciente perder o enxerto precocemente e/ou maior o risco de mortalidade após o transplante hepático..$^{9,10}$

Cerca de $50 \%$ dos pacientes com cirrose hepática apresentam concentrações séricas de sódio inferiores ao intervalo normal $(<135 \mathrm{mEq} / \mathrm{L}) .{ }^{9}$ No entanto, muitos estudos ${ }^{10,11}$ adotam o ponto de corte de sódio sérico $<130 \mathrm{mEq} / \mathrm{L}$.

A relação da hiponatremia pré-operatória e seus fatores prognósticos e interferência na sobrevida pósoperatória ainda é debatida, pois há tantos estudos relatando associação significativa quanto aqueles que não encontraram impacto estatístico. ${ }^{9}$
Foi no sentido de contribuir para a implementação de estratégias de rastreio de fatores prognósticos no póstransplante hepático que esta pesquisa foi desenhada.

O presente trabalho avaliou o MELD-Na e a natremia por meio da análise retrospectiva e associação de parâmetros clínicos-objetivos.

\section{MÉTODOS}

Trata-se de estudo de análise longitudinal retrospectiva, cuja amostra foi composta por 148 prontuários de pacientes que realizaram transplante hepático no Hospital Angelina Caron, entre as datas de 01/01/2016 e 31/12/17, com acompanhamento médico, durante um ano, após a data do transplante.

Foram incluídos pacientes que realizaram transplante de fígado e deram continuidade ao tratamento no seguimento ambulatorial do Hospital Angelina Caron, de Campina Grande do Sul, Paraná. O instrumento utilizado na pesquisa foi baseado na análise de dados dos prontuários.

Foram extraídos dados, como identificação do paciente (idade, sexo designado ao nascimento e cor), para analisar e interpretar o perfil demográfico, comorbidades associadas e sorologias para analisar e interpretar as etiologias que levaram à doença hepática, exames laboratoriais pré-transplante (creatinina sérica, bilirrubina total, RNI e sódio sérico), intercorrências na cirurgia e complicações precoces (até 30 dias) e variáveis relativas aos desfechos pós-transplante (seguimento, sobrevida e/ou retransplante).

O MELD-Na, além dos demais dados laboratoriais, foram obtidos a partir dos dados registrados na última coleta pré-transplante disponível no Sistema Nacional de Transplantes (SNT), sistema responsável pela alocação do órgão para paciente inscrito no processo.

A análise da taxa de sobrevida foi dividida em 3, 6, 12 e 24 meses, após a data inicial do transplante hepático. Para isso, foi utilizado o estimador de Kaplan-Meier e o modelo de riscos proporcionais de Cox; ambos são largamente utilizados em estudos clínicos e consideram tanto os intervalos de tempo, quanto o número de tempos distintos de falha. Também avaliam a suposição de taxa de falhas proporcionais, que serão devidamente ajustadas. Foram estimadas as curvas de sobrevida associadas às categorias de cada covariável, a fim de investigar quais covariáveis estariam associadas ao tempo de sobrevida dos pacientes e quais seriam consideradas candidatas ao modelo de Cox. 
Carolina Oliveira de Paulo, Luize Kremer Gamba, Alice Ferreira da Silva, Carla Martinez, Menini-Stalhschmidt, João Eduardo Leal Nicoluzzi, Matheus Takahashi Garcia

Os resultados de variáveis quantitativas foram descritos por médias, desvios padrões, valores mínimos e máximos. Para variáveis categóricas, foram apresentadas frequências e percentuais. Para comparação de dois grupos, em relação ao tempo em lista de espera, foi usado o teste não-paramétrico de MannWhitney. Mais de dois grupos foram comparados usandose o teste não-paramétrico de Kruskal-Wallis. A condição de normalidade das variáveis quantitativas contínuas foi avaliada pelo teste de Kolmogorov-Smirnov. Para a análise univariada e multivariada de fatores associados ao tempo de sobrevida, foram ajustados modelos de Regressão de Cox e estimados os valores de hazard ratio (HR) com respectivos intervalos de confiança de 95\%. A variável complicação tardia foi incluída nos modelos como tempo-dependente. Para descrição da sobrevida, foram apresentadas curvas de Kaplan-Meier. Valores de $p<0,05$ indicaram significância estatística (ausência de erro aleatório). Os dados foram analisados com o programa computacional Stata/SE v.14.1. StataCorpLP, USA.

Esta pesquisa faz parte do projeto de origem "Perfil epidemiológico e análise da sobrevida de pacientes submetidos ao transplante hepático", foi submetida em junho de 2018 na Plataforma Brasil e aprovada pelo Comite de Ética do Hospital Angelina Caron (CEPHAC) sob o CAAE 91928418.1.0000.5226 e Número do Parecer 2.749.478.

\section{RESULTADOS}

A análise apresentada foi realizada com base nos dados de 148 pacientes submetidos a transplante hepático, no período de janeiro/2016 a dezembro/2017 e acompanhados clinicamente durante o ano de 2018, no Hospital Angelina Caron, localizado no município de Campina Grande do Sul, Paraná, Brasil.

Em relação às variáveis demográficas dos pacientes do estudo ( $n=148)$, foram encontradas $n=105$ pessoas pertencentes ao sexo masculino, com predomínio da cor autodeclarada branca $(n=139)$. A média de idade foi 52 anos $\pm 11,7(20-73)$ e $n=46$ pessoas tinham $\geq 60$ anos. O tempo em lista de espera para o transplante hepático foi de 68 dias \pm 113 ( 1 - 715).

Em relação às variáveis relativas às doenças hepáticas dos pacientes do estudo $(n=148), n=18$ pessoas tinham diagnóstico de hepatite $B$ e $n=14$ pessoas de hepatite C. Foram encontrados $n=50$ indivíduos com cirrose alcoólica, $n=18$ com cirrose autoimune, $n=15$ com cirrose criptogênica, $\mathrm{n}=19$ pacientes tinham hepatocarcinoma
$>2 \mathrm{~cm}, \mathrm{n}=10$ esteatohepatite não alcoólica e $\mathrm{n}=4$ foram classificados como "outros".

O escore MELD-Na obteve média de 13,8 \pm 5,31 (6 39). Os pacientes foram subdividos em MELD-Na $<15$ $(n=105)$ e $>15(n=43)$ e em MELD-Na $<20(n=127)$ e $>20$ $(n=21)$. Em relação à natremia, foi encontrado o valor de sódio sérico $<135$ em 33 pacientes.

A tabela 1 indica as intercorrências intraoperatórias e complicações precoces, após o transplante hepático dos pacientes do estudo.

Tabela 1: Intercorrência intraoperatórias e Complicações precoces $(n=148)$

\begin{tabular}{|c|c|c|}
\hline Variável & Classif. & Resultado* \\
\hline \multirow[t]{2}{*}{ Intercorrência hemodinâmica } & Não & $108(73,0)$ \\
\hline & Sim & $40(27,0)$ \\
\hline \multirow[t]{2}{*}{ Intercorrência vascular } & Não & $136(91,9)$ \\
\hline & Sim & $12(8,1)$ \\
\hline \multirow[t]{2}{*}{ Complicação hemodinâmica } & Não & $127(85,8)$ \\
\hline & Sim & $21(14,2)$ \\
\hline \multirow[t]{2}{*}{ Complicação renal } & Não & $130(87,8)$ \\
\hline & Sim & $18(12,2)$ \\
\hline \multirow[t]{2}{*}{ Complicação vascular } & Não & $139(93,9)$ \\
\hline & Sim & $9(6,1)$ \\
\hline \multirow[t]{2}{*}{ Complicação imunossupressão } & Não & $143(96,6)$ \\
\hline & Sim & $5(3,4)$ \\
\hline \multirow[t]{2}{*}{ Complicação biliar } & Não & $130(87,8)$ \\
\hline & Sim & $18(12,2)$ \\
\hline \multirow[t]{2}{*}{ Rejeição do enxerto } & Não & $139(93,9)$ \\
\hline & Sim & $9(6,1)$ \\
\hline \multirow[t]{2}{*}{ Complicação infecciosa } & Não & $131(88,5)$ \\
\hline & Sim & $17(11,5)$ \\
\hline \multirow{2}{*}{$\begin{array}{l}\text { Intervalo entre transplante e } \\
\text { complicação precoce (dias) }\end{array}$} & Não & \multirow[t]{2}{*}{$6,8 \pm 6,3(1-27)$} \\
\hline & Sim & \\
\hline
\end{tabular}

*Descrito por média \pm desvio padrão (mínimo - máximo)

Em relação às variáveis relativas ao desfecho dos pacientes da amostra, $n=64(43,2 \%)$ vieram à óbito pós-transplante hepático, com média de sobrevida 
de cinco meses $\pm 7,27(0-30,4)$. Ao contrário, aqueles que sobreviveram após o transplante $(n=84)$, a média de seguimento foi de 24,9 meses $\pm 7,7(2,2-49,4)$. $O$ valor médio geral do acompanhamento dos pacientes $(n=148)$ foi de 16,3 meses $\pm 12,4(0-49,4)$. Enfim, cinco pacientes foram submetidos a retransplante, e o tempo de espera na relista foi de 2,6 dias $\pm 2,1$ (1 6 ), enquanto, entre os transplantes, foi de 91,8 dias $\pm 145,8$ (3 - 341).

$\mathrm{Na}$ tabela 2, foram apresentados os percentuais gerais de sobrevida estimados pelo método de Kaplan-Meier. Todos os tempos de seguimento foram considerados. Para pacientes que faleceram, é o tempo até o óbito (sobrevida) e para pacientes que não morreram é o tempo até o último acompanhamento clínico.

Tabela 2: Percentuais gerais de sobrevida $(n=148)$

\begin{tabular}{cc}
\hline Tempo & \% de sobrevida \\
\hline Transplante & $98,6 \%$ \\
1 mês & $81,1 \%$ \\
2 meses & $75,7 \%$ \\
3 meses & $74,3 \%$ \\
4 meses & $72,3 \%$ \\
5 meses & $70,2 \%$ \\
6 meses & $68,9 \%$ \\
12 meses & $61,4 \%$ \\
\hline
\end{tabular}

A figura 1 exibe a curva de sobrevida da amostra total o estudo, com intervalos de confiança de $95 \%$.

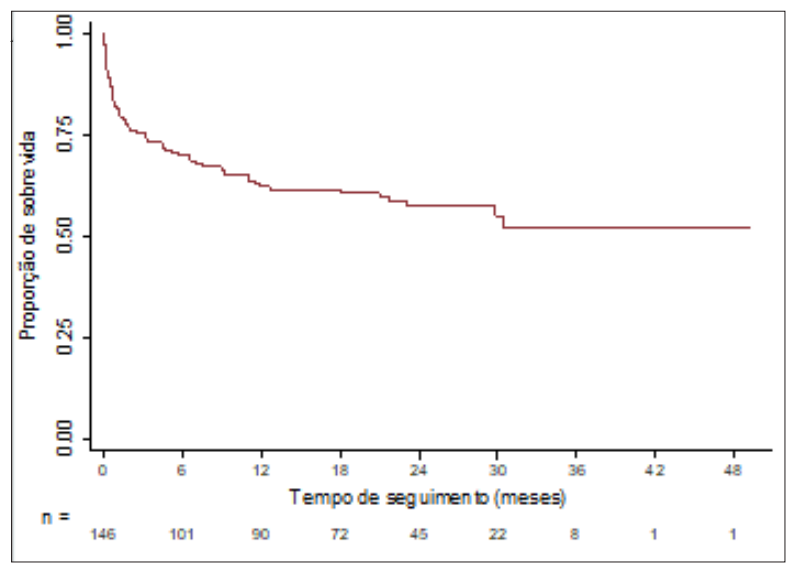

Para avaliar se a presença de hiponatremia antes do transplante estava associada a um risco aumentado de complicações após o transplante, os pacientes foram divididos de acordo com a presença ou ausência de hiponatremia no transplante, e as taxas de complicações entre os dois grupos foram comparadas. Pacientes com hiponatremia apresentaram maior risco de desenvolver complicações após o transplante do que pacientes sem hiponatremia, sendo a diferença no nível de significância.

Utilizando o ponto de corte de $\mathrm{Na}<135 \mathrm{mEq} / \mathrm{L}$ para hiponatremia, a hiponatremia pré-operatória foi inserida no modelo de riscos proporcionais de Cox para mortalidade em 3, 6, 12, 24 e 36 meses, após a data inicial do transplante hepático. Foram analisadas as frequências e os percentuais de casos de óbitos em cada período, de acordo com a presença ou ausência de hiponatremia.

Do total de pacientes com hiponatremia pré-operatória $(n=33), 13$ vieram a óbito em um período após três meses da data do transplante hepático, um em seis meses e cinco em 12 meses. Foi feita comparação estatística dessa taxa de óbito $(12,8 \%)$ com o modelo de regressão de Cox e teste de Wald, cujo valor de $p<0,05$ e HR (IC95\%) estava entre 0,99 a 2,98.

A figura 2 exibe a curva de sobrevida dos pacientes do estudo, de acordo com a hiponatremia ou normonatremia, com intervalos de confiança de $95 \%$.

Figura 2: Curva de sobrevida de acordo com a natremia (n=148)

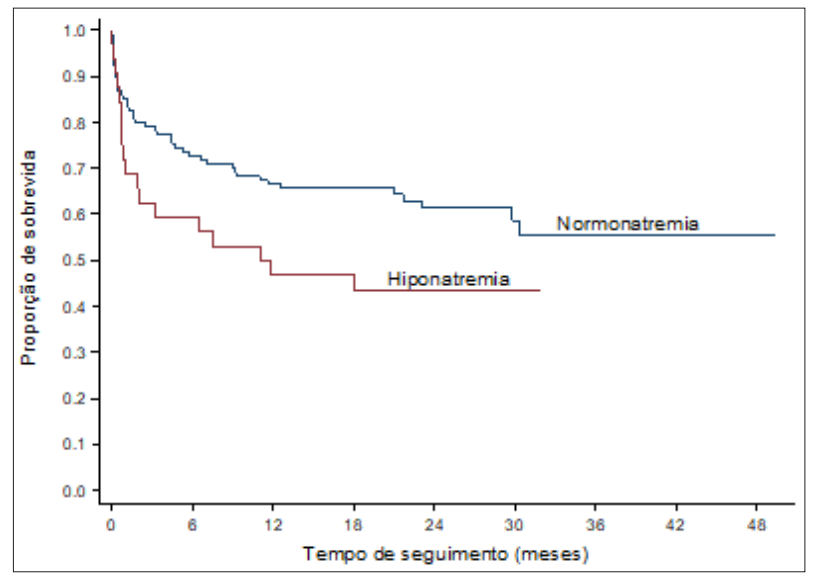

Também foram analisadas as frequências $e$ os percentuais de casos de óbitos, em cada período, de acordo com o escore MELD-Na. Dos pacientes com MELD-Na >15 ( $n=43), 17$ vieram a óbito, dentro de um período de seis meses da data do transplante, e três em 12 meses. Quando o valor MELD-Na aumenta para $>20(n=21)$, observa-se que 10 pacientes vieram à óbito em seis meses e um em 12 meses pós-transplante hepático. Realizando a comparação estatística dessa taxa de óbito $(14,1 \%)$ com o modelo de regressão de Cox e teste de Wald, encontramos um valor de $p<0,05$ e HR (IC95\%) entre 1,57 a 12,4, o que indica ausência de erro sistemático (estudo válido/acurado) e ausência de erro aleatório (estudo confiável/preciso).

Testou-se a hipótese nula de que as distribuições sobre as classificações de MELD-Na são iguais para os 
casos com e sem natremia versus a hipótese alternativa de distribuições diferentes. Na tabela abaixo, são apresentadas estatísticas descritivas e o valor de p. Em ambas análises estatísticas, o p-valor foi <0,001 (ausência de erro aleatório - estudo confiável/preciso).

Tabela 3: Relação entre natremia e MELD-Na

\begin{tabular}{|c|c|c|}
\hline \multirow{2}{*}{ MELD-Na } & \multicolumn{2}{|c|}{ Natremia } \\
\hline & Não & Sim \\
\hline \multirow{2}{*}{$<15$} & 97 & 8 \\
\hline & $84,3 \%$ & $24,2 \%$ \\
\hline \multirow{2}{*}{$>15$} & 18 & 25 \\
\hline & $15,7 \%$ & \\
\hline Total & 115 & 33 \\
\hline \multicolumn{3}{|c|}{ Valor de $\mathrm{p}:<0,001$ (teste de Qui-quadrado, $\mathrm{p}<0,05$ ) } \\
\hline$<20$ & $\begin{array}{c}110 \\
95,7 \%\end{array}$ & $\begin{array}{c}17 \\
51.5 \%\end{array}$ \\
\hline \multirow{2}{*}{$>20$} & 5 & 16 \\
\hline & $4,3 \%$ & 48,4 \\
\hline Total & 115 & 33 \\
\hline \multicolumn{3}{|c|}{ Valor de $\mathrm{p}:<0,001$ (teste exato de Fisher, $\mathrm{p}<0,05$ ) } \\
\hline
\end{tabular}

\section{DISCUSSÃO}

Foram registradas complicações precoces hemodinâmicas $(14,2 \%)$, renais $(12,2 \%)$, inclusive rejeição precoce do enxerto $(6,1 \%)$. A taxa de sobrevida anual da amostra geral desta pesquisa foi de $61,4 \%$, valor semelhante ao da média geral nacional (67\%), de acordo com o Registro Brasileiro de Transplantes (RBT) no biênio 2016-2017.

Na Europa, a taxa de sobrevida anual do paciente de acordo com o European Liver Transplant Registries (ELTR) foi estimada em 90\% no ano de 2018. Nos EUA, o Organ Procurement and Transplantation Network (OPTN) encontrou taxa de sobrevida do paciente de $88 \%$ no ano de 2018. Nesse sentido, a taxa de sobrevivência decresce com o tempo, com probabilidade de sobrevida menor, à medida em que aumenta o intervalo de acompanhamento do paciente após realização da cirurgia.

Já é consenso na literatura médica que a hiponatremia pré-operatória afeta adversamente a sobrevida de pacientes hepáticos em estágio terminal, incluindo aqueles em lista de espera do transplante hepático.
Assim, quanto maior o valor do MELD-Na do paciente, maior é o risco de óbito, teoricamente, e maior é a prioridade na lista de transplante hepático, caso o paciente apresente os requisitos e condições clínicas para tal.

Nosso estudo avaliou o impacto da adoção do sódio sérico o escore MELD, demonstrando que o escore MELD-Na $>15(n=43)$ foi preditor significativo de óbito $(p<0,05)$. Em comparação com outro estudo, ${ }^{10}$ um escore MELD-Na = $20(n=753)$ foi associado a uma taxa de óbito de $6 \%$, dentro de seis meses $(p<0,05)$, enquanto que um escore MELD-Na $=30 \quad(n=753)$ foi associado a um risco de óbito de 16\% $(p<0,05)$. No entanto, não foi analisado o impacto ao critério classificatório para colocação dos pacientes na lista de espera.

Muitos estudos usam $\mathrm{Na}<130 \mathrm{mEq} / \mathrm{L}$ para definir hiponatremia, ${ }^{11,12}$ enquanto outros usam $\mathrm{Na}<135 \mathrm{mEq} / \mathrm{L}$ como ponto de corte,13,14 Neste estudo, a hipótese de que a hiponatremia $(\mathrm{Na}<135 \mathrm{mEq} / \mathrm{L})$ pré-operatória estaria associada a uma maior chance de mortalidade após o transplante hepático foi comprovada mediante análises estatísticas, com taxa de óbito de 12,8\% e valor de $p<0,05$. Resultado semelhante foi visto em outros dois estudos, em que, em uma amostra de $n=753$ pacientes ,houve taxa de óbito de $17 \%$ e $p<0,05$, em um intervalo de tempo, após seis meses da data do transplante ${ }^{10} \mathrm{e}$, em outra amostra de $\mathrm{n}=241$ pacientes, houve uma taxa de óbito de $16 \%$ e $p<0,05$, após três meses do transplante hepático. ${ }^{10}$

Em um estudo, ${ }^{15}$ foi visto que queda de $1 \mathrm{mEq} / \mathrm{L}$ do sódio sérico (quando inferior a $135 \mathrm{mEq} / \mathrm{L}$ ) reduziu $12 \%$ da taxa de sobrevida dos pacientes avaliados, num período de três meses. Em outro, ${ }^{16}$ observou-se que o efeito da hiponatremia pré-operatória na lista de espera diminui gradualmente a taxa de óbito, à medida que o escore MELD-Na aumenta, reduzindo a mortalidade na lista de espera, em até $7 \%$.

A maioria dos pacientes com doença hepática grave, principalmente aqueles com cirrose descompensada, apresenta hiponatremia hipervolêmica crônica. A restrição diurética é útil, no entanto, aumenta os níveis séricos de sódio, o que contribui para a piora da sobrecarga de líquidos. ${ }^{17}$

É importante ressaltar a fisiopatologia decorrente de um valor de sódio sérico baixo, pois a hiponatremia crônica está associada à retenção de água livre de soluto (aumento da secreção do $\mathrm{ADH}$ ), elevando o risco de ascite, síndrome hepatorrenal e agravando a ocorrência da hipertensão portal em pacientes com cirrose prévia..$^{18,19}$ 
Neste estudo, houve limitações como pacientes de um único centro - viés de pesquisa e a não-análise do impacto como critério classificatório para colocação dos pacientes na lista de espera. Ademais, na maioria das pesquisas, inclusive neste estudo, a natremia pré-operatória foi obtida em um único momento, com intervalo variado até o momento do transplante. Por fim, gostaríamos de propor uma intervenção antes do transplante, como corrigir o valor sérico de sódio antes do transplante, já que apresentam uma sobrevida muito ruim.

\section{CONCLUSÃO}

A análise estatística demonstrou a hiponatremia como preditor significativo de risco de mortalidade no póstransplante hepático. Apesar das limitações, sugerimos que a vigilância da hiponatremia pré-operatória, o cálculo do escore MELD-Na, bem como o impacto na classificação da lista de espera dos pacientes prétransplante hepático sejam fortemente incentivados, como forma de redução da taxa de mortalidade póstransplante hepático no Brasil.

\section{ABSTRACT}

Introduction: Brazil is the largest public transplant system in the world. Recently, the MELD-Sodium score was incorporated in Brazil as a model to predict severity of liver disease. In international studies, it was observed that below normal serum sodium values are associated to graft loss. However, its relationship with prognostic factors in postoperative survival is still uncertain. Purposes: To contribute to implement screening prognostic factor strategies in post-liver transplantation. Methods: Retrospective longitudinal study including patients who underwent liver transplantation between the dates 01/01/2016 and 12/31/17, and who had one-year medical follow-up after the date of the transplant. Results: Final sample of 148 patients, from whom $n=105$ were male with mean age of 52 years \pm 11.7 and $n=139$, and declared themselves as white. $N=50$ individuals were bearer of alcoholic cirrhosis, and $n=32$ viral hepatitis. The time on the waiting list for liver transplant was 68 days \pm 113 . The average MELD score was 20.7 \pm 5.56 , while MELD-Na was 13.8 \pm 5.31 . MELD-Na values $<15(n=105)$, MELD-Na $>15(n=43)$ were found. The overall 6 -month survival rate was $68.9 \%$ in $61.4 \%$ at 12 months and $56.7 \%$ at 24 months. Preoperative hyponatremia $<135$ $\mathrm{mEq} / \mathrm{L}$ ) present in 33 patients, in which $\mathrm{n}=14$ died within six post-transplant months, $\mathrm{n}=4$ in 12 months, and $\mathrm{n}=1$ in 24 months. The analysis between hyponatremia and death obtained $p<0,05$. MELD-Na $>15(n=43)$, from which $n=17$ died within six post-transplant months, $n=3$ in 12 months and $n=1$ in 24 months. Upon assessing the MELD score, values $>20(n=89)$ were significant predictors of death $(p<0.05)$, adjusting it for the MELD-Na score, values $>15(n=43)$ were significant predictors of death $(p<0.05)$. Conclusion: The monitoring of preoperative hyponatremia and the use of MELD-Na to define priority as to procedure on the waiting list should be strongly encouraged as a way to reduce post-liver transplant mortality rate.

Keywords: Liver transplantation, Hyponatremia, Survival, Mortality, Prognosis 


\section{REFERÊNCIAS}

1. National Institutes of Health Consensus Development Conference Statement. Liver Transplantation. Hepatology 1984;4(1):107S-110S.

2. D'Albuquerque LA, de Oliveira e Silva A. Transplante de Fígado. Arq. Gastroenterol. 1993;30(4):79-81.

3. Dopazo C, Bilbao I, Castells LL, Sapisochin G, Moreiras C, Campos-Varela I, et al. Analysis of adult 20-year survivors after liver transplantation. Hepatol. Int. 2015;9(3):461-70.

4. Castro-e-Silva Jr O de, Sankarankutty AK, Oliveira GR de, Pacheco E, Ramalho FS, Sasso KD, et al. Transplante de fígado: indicação e sobrevida. Acta Cir. Bras. 2002;17(Suppl 3):83-91.

5. Fernandes J, Millan L, Massarollo PC, Mies S. Efeito da escala MELD na mortalidade após transplante de fígado. Rev. Med. 2002;81(1-4):15-21.

6. Abdalla S, Alves R, Fonseca E, Vicenzi R, Gonçalves $\mathrm{J}$, Waisberg J. A hiponatremia como fator preditivo da perda precoce do transplante hepático na modalidade intervivos. Arq. Bras. Ciências da Saúde 2012;37(2):7680.

7. Kamath PS, Wiesner RH, Malinchoc M, Kremers W, Therneau TM, Kosberg CL, et al. A model to predict survival in patients with end-stage liver disease. Hepatology 2001;33(2):464-70.

8. Liver and Intestinal Organ Transplanation Committee - OPTN/UNOS. Clerical hanges for implementation of adding serum sodium to the MELD Score. 2015.

9. Yang S-M, Choi S-N, Yu JH, Yoon H-K, Kim WH, Jung $\mathrm{C}-\mathrm{W}$, et al. Intraoperative hyponatremia is an independent predictor of one-year mortality after liver transplantation. Sci. Rep. 2018;8(1):1-9.

10. Biggins SW, Kim WR, Terrault NA, Saab S, Balan V, Schiano T, et al. Evidence-based incorporation of serum sodium concentration into MELD. Gastroenterology 2006;130(6):1652-60.
11. Londono M-C, Guevara M, Rimola A, Navasa M, Taura P, Mas A, et al. Hyponatremia impairs early posttransplantation outcome in patients with cirrhosis undergoing liver transplantation. Gastroenterology 2006;130(4):1135-43.

12. Dawwas MF, Lewsey JD, Neuberger JM, Gimson AE. The impact of serum sodium concentration on mortality after liver transplantation: a cohort multicenter study. Liver Transplant. 2007;13(8):1115-24.

13. Wang P, Huang G, Tam N, Wu C, Fu S, Hughes BP, et al. Influence of preoperative sodium concentration on outcome of patients with hepatitis B virus cirrhosis after liver transplantation. Eur. J. Gastroenterol. Hepatol. 2016;28(10):1210-5.

14. Yun BC, Kim WR, Benson JT, Biggins SW, Therneau TM, Kremers WK, et al. Impact of pretransplant hyponatremia on outcome following liver transplantation. Hepatology 2009;49(5):1610-5.

15. Londono M-C, Cardenas A, Guevara M, Quinto L, de Las Heras D, Navasa M, et al. MELD score and serum sodium in the prediction of survival of patients with cirrhosis awaiting liver transplantation. Gut 2007;56(9):1283-90.

16. Kim WR, Biggins SW, Kremers WK, Wiesner RH, Kamath PS, Benson JT, et al. Hyponatremia and mortality among patients on the liver-transplant waiting list. N. Engl. J. Med. 2008;359(10):1018-26.

17. Leise M, Cardenas A. Hyponatremia in Cirrhosis: Implications for Liver Transplantation. Liver Transplant. Off. Publ. Am. Assoc. Study Liver Dis. Int. Liver Transplant. Soc. 2018;24(11):1612-21.

18. Biggins SW, Rodriguez HJ, Bacchetti P, Bass NM, Roberts JP, Terrault NA. Serum sodium predicts mortality in patients listed for liver transplantation. Hepatology 2005;41(1):32-9.

19. Machicao VI. Model for End-Stage Liver DiseaseSodium Score: The Evolution in the Prioritization of Liver Transplantation. Clin. Liver Dis. 2017;21(2):275-87. 